\title{
Chaotic difference equations are dense
}

\section{Peter E. Kloeden}

A continuous function mapping a compact interval of the real line into itself is called chaotic if the difference equation defined in terms of it behaves chaotically in the sense of Li and Yorke. The set of all such chaotic functions is shown to be a dense subset of the space of continuous mappings of that interval into itself with the $\max$ norm. This result indicates the structural instability of nonchaotic difference equations with respect to chaotic behaviour.

\section{Introduction}

Numerical studies of the solutions of one-dimensional deterministic difference equations

$$
x_{n+1}=f\left(x_{n}\right), n=0,1,2, \ldots,
$$

describing meteorological $([7,8])$ and biological $([10,11])$ phenomena have indicated behaviour so apparently irregular that it might well be termed chrotic. In a recent paper $L i$ and Yorke [6] clearly specified what might heuristically be thought of as chaotic behaviour in a difference equation (1.1) and, indeed, it was they who suggested the term chaos to describe such behaviour.

In this paper functions $f$ in difference equation (1.1) will be considered which are continuous and map a compact interval $X$ of the real line into itself. The space of all such functions with the $\max$ norm will be denoted by $\mathcal{C}(X)$ and any such function for which difference equation Received 9 July 1976. 
(1.1) behaves chaotically in the sense of $\mathrm{Li}$ and Yorke will be called chaotic. It will be proved that the subset of all such chaotic functions is a dense subset of $c(X)$. This will be done by actually constructing for each function $f$ in $C(X)$ and each desired degree of accuracy $\varepsilon>0$, a chaotic function in $C(X)$ which lies within $\varepsilon$ of $f$ with respect to the $\max$ norm on $\mathcal{C}(X)$.

Although this result suggests the structural instability with respect to chaotic behaviour of nonchaotic difference equations, it is not quite so serious as it may at first seem on account of the localised nature of the chaotic behaviour in the constructed function. It does, however, indicate the need for some kind of measure of the extent of chaotic behaviour and the need to restrict a discussion of such structural instability to parametric perturbations rather than arbitrary perturbations.

\section{Chaotic difference equations}

Let $X=[a, b]$ be a compact interval and let $\mathcal{C}(X)$ denote the space of all continuous functions $f: X \rightarrow X$ with the max norm $\|f\|=\max _{x \in X}|f(x)|$.

Associated with each $f$ in $\mathcal{C}(X)$ there is a difference equation

$$
x_{n+1}=f\left(x_{n}\right), n=0,1,2, \ldots,
$$

for which a set of $k=1,2,3, \ldots$ distinct points $p_{0}, p_{1}, \ldots, p_{k-1}$ is called a cycle of order $k$ if $p_{i}=f\left(p_{i-1}\right)$ for $i=1,2, \ldots, k-1$ and $p_{0}=f\left(p_{k-1}\right)$. Note that cyclic points of any order $k$ are fixed points of the kth-iterate $f^{k}=f \circ f \circ \ldots \circ f(k$ times) of $f$ and that each $f$ in $\mathcal{C}(X)$ has at least one fixed point (of order 1 ).

For a given difference equation (2.1) an initial point $x_{0}$ in $X$ is said to be asymptotically periodic if there exists a cyclic point $p_{0}$ of some order such that

$$
\lim _{n \rightarrow \infty}\left|f^{n}\left(x_{0}\right)-f^{n}\left(p_{0}\right)\right|=0 .
$$

This represents a highly desirable form of behaviour for all points 
$x_{0}$ in $X$ and their associated iterative sequences. Coppel [5] has shown this to be the case for difference equations having cycles of only finitely many different orders (necessarily all of the form $2^{n}$ ), though the following theorem of $\mathrm{Li}$ and Yorke [6] shows that it is not obtainable in all difference equations.

THEOREM 1. Suppose for $f$ in $\mathcal{C}(X)$ that the difference equation (2.1) has a cycle of order three. Then it has cycles of all orders and there exists an uncountable subset $S$ of $X$ containing no cyclic points such that for all $x_{0}, y_{0}$ in $S$ and all cyclic points $p_{0}$ of any order the following hold:

(i) $\lim _{n \rightarrow \infty} \sup \left|f^{n}\left(x_{0}\right)-f^{n}\left(y_{0}\right)\right|>0$,

(ii) $\lim _{n \rightarrow \infty} \inf \left|f^{n}\left(x_{0}\right)-f^{n}\left(y_{0}\right)\right|=0$, and

(iii) $\lim _{n \rightarrow \infty} \sup \left|f^{n}\left(x_{0}\right)-f^{n}\left(p_{0}\right)\right|>0$.

This behaviour was described quite aptly by $L i$ and Yorke as being chaotic.

Actually related and more powerful results appeared earlier in the Eastern European mathematical literature (for example, [1, 2, 3, 4, 12 , 13]). In particular, the work of the Ukrainian mathematician A.N.

Sarkovski $Y$ (for example, $[12,13]$ ) lead Kloeden, Deakin, Tirkel [9] to weaken the conclusions of Theorem 1 above in defining chaotic behaviour to allow the absence of some (in fact, possibly infinitely many) cyclic orders, provided that there is always at least one cycle present with order of the form $(2 k+1) .2^{n}$ for some nonzero $k$. In this paper, however, the more restrictive definition of $L i$ and Yorke will be used and any $f$ in $\mathcal{C}(X)$ with difference equation (2.1) behaving in such a manner will be called chaotic.

Examples of chaotic functions occurring in meteorological and biological modelling are given in $[7,8,10,11]$. Other examples can be found in the papers cited above. The following example is a chaotic function and will be used in proving Theorem 2 . 
EXAMPLE 1. Let $\eta>0$ and let $\Lambda \in \mathcal{C}([0,2 \eta])$ be defined as

$$
\Lambda(x)= \begin{cases}2 x & \text { for } 0 \leq x \leq n, \\ -2 x+4 n & \text { for } \eta \leq x \leq 2 \eta .\end{cases}
$$

This has a cycle of order three for any value of $\eta>0$, namely $p_{0}=\frac{4}{7} n, p_{1}=\frac{8}{7} n, p_{2}=\frac{12}{7} n$, and by Theorem.1, is thus a chaotic function. Its maximum value is $2 n$ and occurs at $x=n$. It is illustrated together with its $n$ th-iterates $\Lambda^{n}$ in Figure 4 of [1] for $n=\frac{1}{2}$.

\section{Main result}

The following theorem is the main result of this paper. Recall that a subset of a topological space is dense if its closure is equal to the whole space.

THEOREM 2. The chaotic functions are dense in $\mathrm{C}(X)$.

Altermatively, for any $f \in \mathcal{C}(X)$ and any $\varepsilon>0$ there is a chaotic function $g \in \mathcal{C}(X)$ such that

$$
\|f-g\|<\varepsilon .
$$

Proof. Let $f \in \mathcal{C}(X)$ and $\varepsilon>0$ be given, but otherwise arbitrary. The theorem will be proved by constructing a chaotic function $g \in C(X)$ for which inequality (3.1) is satisfied.

Let $x^{*}$ be a fixed point of $f$ and assume for the present that it is not equal to $b$. Then as $f$ is continuous at $x^{*}$ there is a $\delta=\delta\left(x^{*}, \varepsilon\right)>0$, which can be chosen to satisfy $\delta<\varepsilon / 2$, such that

$$
\left|f(x)-x^{*}\right|<\varepsilon / 2
$$

holds for all $x \in\left[x^{*}, x^{*}+\delta\right]$.

Let $\eta=\delta / 4$ and define $\Lambda^{*} \in C\left(\left[x^{*}, x^{*}+2 \eta\right]\right)$ as

$$
\Lambda^{*}(x)= \begin{cases}2 x-x^{*} & \text { for } x^{*} \leq x \leq x^{*}+\eta, \\ -2 x+3 x^{*}+4 \eta & \text { for } x^{*}+\eta \leq x \leq x^{*}+2 \eta,\end{cases}
$$

which has the same graph as function $\Lambda$ of Example 1, but with the origin 
translated to the point $\left(-x^{*},-x^{*}\right)$.

Also, let $\gamma:\left[x^{*}+2 n, x^{*}+3 n\right] \rightarrow X$ be defined as

$$
\gamma(x)=x^{*}+\left(x-x^{*}-2 \eta\right)\left(\frac{f\left(x^{*}+3 \eta\right)-x^{*}}{\eta}\right),
$$

the graph of which is the straight line segment joining the points $\left(x^{*}+2 n, x^{*}\right)$ and $\left(x^{*}+3 n, f\left(x^{*}+3 n\right)\right)$ in the plane.

Finally, define a function $g: X \rightarrow X$ as

$$
g(x)= \begin{cases}f(x) & \text { for } x \in X \backslash(I \cup J), \\ \Lambda^{*}(x) & \text { for } x \in I \\ \gamma(x) & \text { for } x \in J\end{cases}
$$

where $I=\left[x^{*}, x^{*}+2 \eta\right]$ and $J=\left[x^{*}+2 \eta, x^{*}+3 \eta\right]$.

The graph of such a $g$ is illustrated in Figure I (see page 376). It is obvious that $g$ is a continuous function; that is, $g \in \mathcal{C}(X)$. Note that

$$
|f(x)-g(x)|= \begin{cases}0 & \text { for } x \in X \backslash(I \cup J), \\ \left|f(x)-\Lambda^{*}(x)\right| & \text { for } x \in I, \\ |f(x)-\gamma(x)| & \text { for } x \in J .\end{cases}
$$

Hence

$$
\begin{aligned}
\|f-g\| & =\max _{x \in I}\left|f(x)-\Lambda^{*}(x)\right|, \max _{x \in J}|f(x)-\gamma(x)| \\
& \left.\leq \max _{x \in I}\left|\max _{x \in I}\right| f(x)-x^{*}\left|+\max _{x \in I}\right| x^{*}-\Lambda^{*}(x)\left|, \max _{x \in J}\right| f(x)-x^{*}\left|+\max _{x \in J}\right| x^{*}-\gamma(x) \mid\right\} \\
& <\max \{\varepsilon / 2+\varepsilon / 4, \varepsilon / 2+\varepsilon / 2\} \\
& =\varepsilon
\end{aligned}
$$

on account of inequality (3.2), the definitions of intervals $I$ and $J$, and the two inequalities

$$
\begin{aligned}
& \max _{x \in I}\left|x^{*}-\Lambda^{*}(x)\right|=2 \eta=\delta / 2<\varepsilon / 4, \\
& \max _{x \in J}\left|x^{*}-\gamma(x)\right|=\left|x^{*}-f\left(x^{*}+3 \eta\right)\right|<\varepsilon / 2 .
\end{aligned}
$$

That this $g$ is a chaotic function follows immediately from Theorem I and the fact that $\Lambda^{*}$, and hence $g$ itself, has a cycle of order three, namely $p_{0}=x^{*}+\frac{4}{7} n, p_{1}=x^{*}+\frac{8}{7} n, p_{2}=x^{*}+\frac{12}{7} \eta$. 


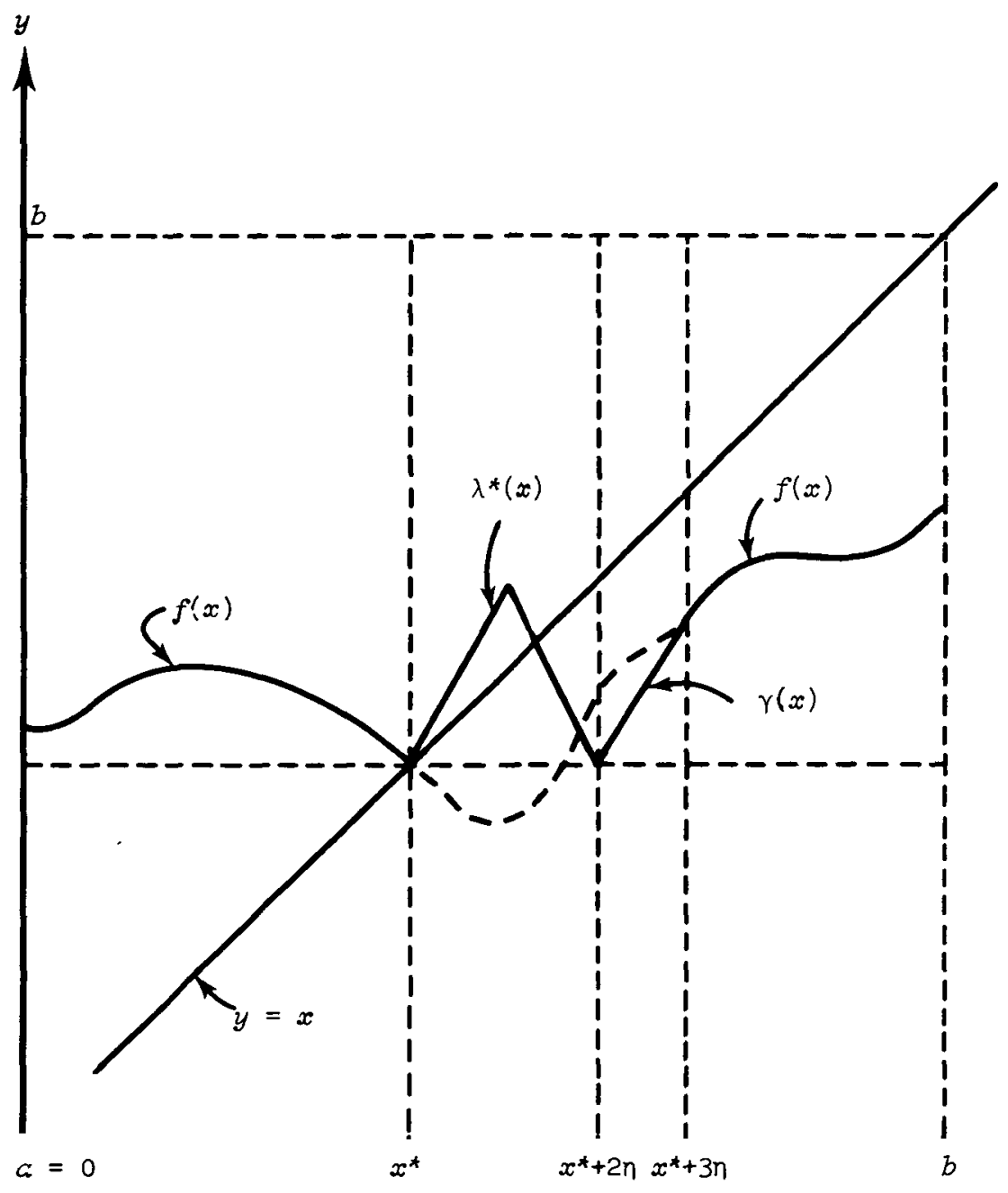

FIGURE 1. Function $g$ when $x^{*} \neq b$.

It now remains to consider the case where the only fixed point of $f$ is larger endpoint $b$ of the interval $X$. Here a chaotic function $g$ satisfying inequality (3.1) can be constructed in the same way as above, except with the $\Lambda^{*}$ perturbation now the reflection in the point $\left(x^{*}, x^{*}\right)$ of that above. This is illustrated in Figure 2.

This completes the proof of the theorem.

REMARK. Since a function which is chrotic in the sense of $\mathrm{Li}$ and Yorke is also chaotic in the weaker sense of Kloeden, Deakin, Tirkel the 
above theorem is also true for this weaker definition of chaotic functions.

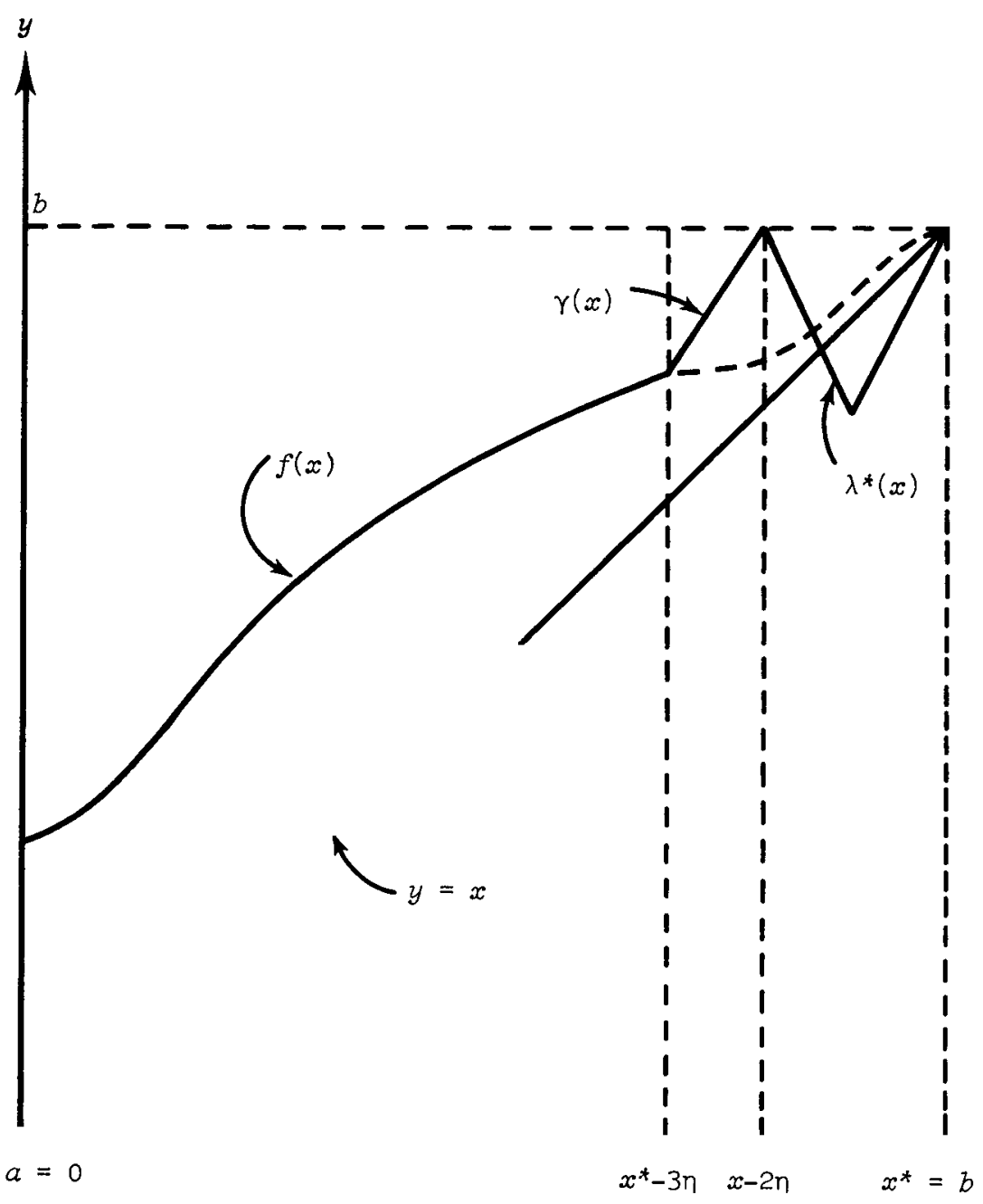

FIGURE 2. Function $g$ when $x^{*}=b$.

\section{Concluding remarks}

Theorem 2 can be interpreted as saying that nonchaotic difference equations are structurally unstable with respect to chaotic behaviour. The proof of the theorem shows, however, that this is not quite so devastating as it may at first seem.

Observe that, for sufficiently small bound $\varepsilon$ on the perturbations, 
it is unlikely, with the perturbations used, that the behaviour of the perturbed difference equation would differ much from that of the original one outside of the interval $\left[x^{*}, x^{*}+3 \eta\right]$ in which the perturbations are made. Indeed the chaotic behaviour of the perturbed difference equation will most likely be restricted entirely to the interval $\left[x^{*}, x^{*}+2 \eta\right]$ in which the perturbation $\Lambda^{*}$ is defined. Iterative sequences entering this interval from outside it will be trapped inside it and may then begin to behave chaotically. In constructing a mathematical model from physical measurements of such behaviour, given the limited accuracy of any physical measurements, this would probably be mistaken for convergence to the fixed point $x^{*}$. This suggests that chaotic functions should be further classified in terms of the size of the region in which their iterative sequences behave chaotically.

A second point is that $\Lambda^{*}$ is not what one would consider as a naturally occurring perturbation. Indeed, in [9] Kloeden, Deakin, Tirkel suggest that a discussion on structural instability of difference equations with respect to chaotic behaviour should be related to parametric perturbations in the functions being considered, rather than a somewhat arbitrary and unnatural perturbation like $\Lambda^{*}$.

\section{References}

[1] Béla Barna, "Über die Iteration reeller Funktionen I", Publ. Math. Debrecen 7 (1960), 16-40.

[2] Béla Barna, "Über die Iteration reeller Funktionen II", Publ. Math. Debrecen 13 (1966), 169-172.

[3] Béla Barna, "Über die Iteration reeller Funktionen III", Publ. Math. Debrecen 22 (1975), 269-278.

[4] Lothar Berg, "Über irregulare Iterationsfolgen", Publ. Math. Debrecen 17 (1970), 111-115.

[5] W.A. Coppel, "The solution of equations by iteration", Proc. Cambridge Phizos. Soc. 51 (1955), 41-43.

[6] Tien-yien Li and James A. Yorke, "Period three implies chaos", Amer. Math. Monthly 82 (1975), 985-992. 
[7] Edward N. Lorenz, "Deterministic nonperiodic flow", J. Atmospheric Sci. 20 (1963), 130-141.

[8] Edward N. Lorenz, "The problem of deducing the climate from the governing equations", Tellus 16 (1964), 1-11.

[9] Peter Kloeden, Michael A.B. Deakin, A. Tirkel, "A precise definition of chaos", Nature (to appear).

[10] Robert M. May, "Biological populations with nonoverlapping generations: stable points, stable cycles, and chaos", Science $186^{\circ}(1974), 645-647$.

[11] Robert M. Day, "Biological populations obeying difference equations: stable points, stable cycles, and chaos", J. Theoret. Biol. 51 (1975), 511-524.

[12] A.H. Шарновский [A.N. ŠarkovskiY̌], "Сосуцествованне цинлов непрерывного преобразования прямой в себя" [Coexistence of cycles of a continuous transformation of a line into itself], Ukrain. Mat. ̌̌. 16 (1964), no. 1, 61-71.

[13] A.H. Шарновсний, [A.N. Śarkovski i], "О цинлах и струнтуре неприрывного отображення" [On cycles and the structure of a continuous transformation], Ukrain. Mat. ¿. 17 (1965), no. 3, 104-111.

Department of Mathematics,

Monash University,

Clayton,

victoria. 